\title{
Comportement de drageons de pommier (Malus pumila Mill.) issus des cultivars Golden Delicious et Stark Golden Spur
}

\author{
Luc DECOURTYE \& Jacques LEMOINE
}

I.N.R.A., Station d'Amélioration des Espèces fruitières et ornementales, Beaucouzé, F 49000 Angers

Mots clés additionnels : Bouturage de racines, stade juvénile, chimère. Golden Spur.

Rooted cuttings of apple cvs Golden Delicious Standard and Stark Golden Spur were grown for several years according to figure 1 . The trees were then cut off at the roots. Neoformed buds developed on the roots and gave new shoots. Five buds of these shoots were then propagated on a clonal root stock (MM 106). The maiden trees which originated from neoformations on Golden Delicious roots ( $R$ in the tables) were all different from the mother variety by common features: increased branching, smaller leaves (at significant level) and taller and thinner habit (table 1). Under cultivation for 5 years, growth, measured by trunk girth and total shoot length, was close to the control (table 3 and fig. 2). Number of flowers per tree remained lower than the control during the first two years (table 4), gradually reaching the control level (fig. 3). Similarly, leaf areas increased progressively to the control size (fig. 3). According to Zimmerman's scheme on juvenility (1976), this phenomenon can be interpreted as a shifting back, more or less complete, to the juvenile stage of a seedling. The maiden trees which originated from neoformations on Golden Stark Spur roots fell into two patterns. On the one hand, those which originated from $\mathrm{r} 1, \mathrm{r} 3$ and $\mathrm{r} 4$ roots differed strongly from the Stark Golden Spur control by increased growth (trunk girth, number of branches and total shoot length). Due to various diseases (chlorotic leaf spot, stem pitting and rubbery wood), these trees were significantly smaller (10\%) than those of Golden Delicious, but their general habit was of a standard type (fig. 2). These data suggest strongly that the deeper layer LIII of Stark Golden Spur, from which the roots originated, still carrics the genetic standard character of growth. As the spur character has been shown to be transmitted through the gametes (DECOURTYE, 1969), which originated from layer II, the chimeral structure of Stark Golden Spur is thus established. This structure can account for the instability of the clone which occurs in commercial orchards. On the other hand, trees issued from $r 2$ root remained closely similar to the Stark Golden Spur control for most characters, as well for nursery features (table 2), as for orchard ones (1able 3). This $\mathrm{r} 2$ clone has now been made virus- free, which should afford a stable clone of Stark Golden Spur for growers. 


\section{INTRODUCTION}

Beaucoup de cultivars de pommier sont des mutants issus d'une variété préexistante, améliorée généralement pour la coloration du fruit ou l'aspect plus ou moins bronzé de l'épiderme. La plupart de ces mutants sont en chimère, ce qui peut facilement s'observer par des retours à la variété d'origine, avec une fréquence trop élevée pour être une mutation de retour.

Plus récemment, sont apparus sur le marché, parmi les grandes variétés commerciales, des mutants spontanés modifiant à la fois la vigueur et la productivité, les mutants spurs. Ces derniers se caractérisent par des entre-nœuds plus courts avec des productions fruitières localisées à l'extrémité de rameaux courts (brachyblastes), tandis que la variété d'origine, dite standard, fructifie principalement sur des rameaux longs (auxiblastes), aussi bien à partir de bourgeons axillaires que de bourgeons terminaux. En verger, les arboriculteurs constatent fréquemment une hétérogénéité entre ces arbres plus importante que ce qu'ils connaissent habituellement avec la variété d'origine. Observant ainsi trois clones spurs de Golden Delicious, FAEDI \& ROSATI (1985) constatent, dès la première année, que 1 à 15 p. 100 des scions, selon les clones, sont du type standard. Après cinq années, ce taux varie de 4 à 23 p. 100. Cette fréquence élevée de réversion est difficilement compatible avec un taux de mutation de retour et se rapproche davantage de ce que l'on peut observer sur des arbres en chimère.

La contribution des couches successives du méristeme de pommier à l'ontogénèse des différents organes de la plante a été bien établie (TILNEY BASSET, 1986). A partir de chimères polyploüdes de type $2 x-2 x-4 x$, DERMEN (1951) a notamment montré que, dans les tiges, la couche LII constituait préférentiellement les tissus externes à l'assise cambiale (parenchyme et liber), tandis que la couche LIII constituait les tissus internes (xylème et moelle). La ligne de partage entre les contributions de ces deux couches ne se confond pas d'une façon absolue avec l'assise cambiale, de telle sorte que des écarts au schéma décrit peuvent se produire.

Lors du bouturage de la chimère de pommier Giant Spy $2 x-2 x-4 x$, les racines adventives, dont on sait qu'elles prennent naissance au niveau des tissus conducteurs caulinaires (FAVRE, 1977), ont toutes été comptées tétraploïdes (EINSET, 1952). Lorsque nous avons bouturé une chimère anthocyanée de poirier de constitution Rouge-Rouge-Vert, 48 racines sur 50 ont produit des pousses vertes, tandis que les deux autres ont produit des pousses rouges traduisant une contribution exceptionnelle de la LII à la zone cambiale génératrice de ces deux racines (DECOURTYE, 1987).

La couche LII est aussi celle qui est à l'origine des gamètes, de telle sorte que le caractère de polyploïdie n'est pas transmis pour une chimère de type $2 x-2 x-4 x$, alors qu'il l'est pour une chimère de type $2 x-4 x-2 x$ L'étude de l'hérédité du caractère spur a montré que, confrontée à un même parent testeur, une descendance de type spur est quantitativement plus courte qu'une descendance de type standard (DECOURTYE \& LANTIN, 1969). La mutation est donc présente dans la couche de gamétogénèse LII.

L'irradiation aux rayons gamma de bourgeons dormants conduit, par les lésions qu'elle provoque, à la mise en place d'un méristème de cicatrisation susceptible de se constituer à partir de quelques cellules de la couche profonde. Ce processus s'est montré efficace pour séparer les différents tissus constitutifs de chimères colorées d'arbres fruitiers (DECOURTYE, 1969). L'irradiation, dans les mêmes conditions, de différents mutants spurs de Golden Delicious n'a pas montré de retour évident au type standard, n'apportant pas ainsi la preuve du caractère chimérique de ces mutants.

Nous avons alors cherché à accéder à la connaissance génétique de la couche LIII en bouturant ces mutants.

\section{MATÉRIEL ET MÉTHODES}

Deux cultivars de pommier (Malus pumila Mill.) ont été utilisés, Golden Delicious (standard), considéré comme référence et un de ses mutants de type spur, Stark Golden Spur. Le clone de Golden Delicious était sans virus tandis que le clone de Stark Golden Spur s'est révélé contaminé par trois virus, celui des taches chlorotiques du pommier (C.L.S.V.), celui du dépérissement du Spy 227 (S.P.V.) et celui du bois caoutchouc (R.W.V.). A eux seuls, ces virus entraînent une perte de vigueur de l'ordre de 10 p. 100 par rapport au même clone assaini par thermothérapie (LEMOINE, 1986).

Une centaine d'axes feuillés terminaux ont été bouturés sous brouillard, en juin 1974, après trempage de la partie basale dans une poudre contenant 5000 parties par million d'acide indol butyrique. Les taux d'enracinement ont été de l'ordre de 50 p. 100 , mais une mortalité importante au cours de l'hiver suivant a ramené le nombre des boutures survivantes à 12 pour Golden Delicious et à 5 pour Stark Golden Spur. Ces boutures ont été plantées dans un pot de terre de petit volume (20 cm de diamètre), percé de trous latéraux, l'ensemble étant installé dans une bassine de grand diamètre (fig. 1). La nutrition s'effectue par goutteurs de solution nutritive installés à la périphérie de la bassine, de telle sorte que les racines sortent du pot de terre par les trous latéraux et colonisent le substrat extérieur. Lorsque celles-ci ont atteint la dimension souhaitée, il suffit de les sectionner au niveau des orifices en utilisant verticalement une lame de couteau. On sépare ainsi l'arbre avec une motte suffisante pour sa reprise et les racines restent en place dans la grande bassine.

Après plusieurs semaines, apparaissent sur quelques racines, en position subterminale, des excroissances qui finissent par se fendre et laissent apparaître un bourgeon feuillé, rapidement chlorophyllien. Anatomiquement, ces bourgeons adventifs apparaissent au hasard dans le parenchyme cortical et ne se raccordent qu'ultérieurement au cambium vasculaire (ROBINSON \& SCHWABE, 1977). L'hétérogénéité dans les dates d'apparition et dans la nature du système racinaire sous-jacent ne permet pas de juger sur le drageon lui-même des caractéristiques spur ou standard.

Nous avons alors procédé à une multiplication végétative de chaque drageon, considéré comme un sousclone, par écussonnage sur le porte-greffe cloné MM 106, de manière à obtenir au moins cinq scions (= axe 1) du même drageon.

Deux campagnes de greffages ont été nécessaires: la première (1980) a permis de multiplier cinq drageons de 


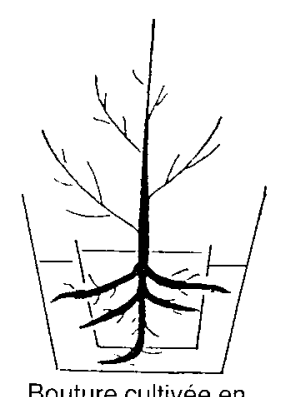

Bouture cultivée en double pot

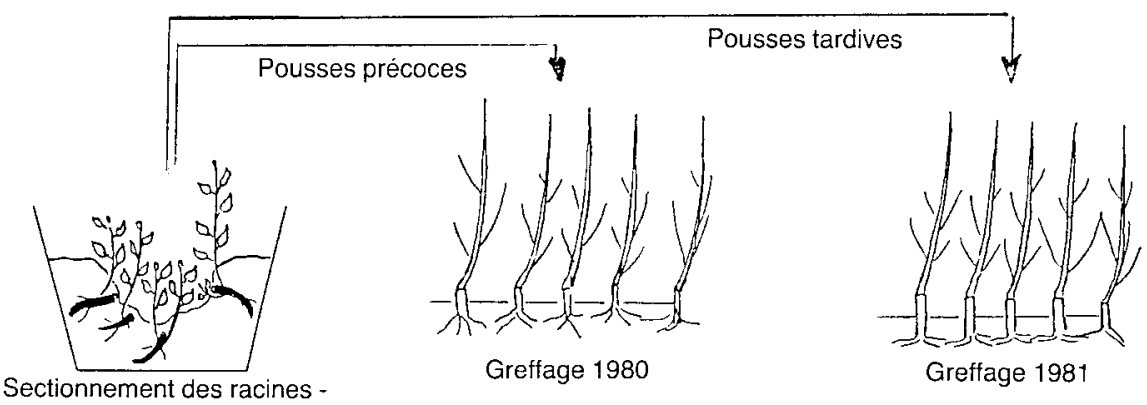

Sectionnement des racines (printemps 1979)
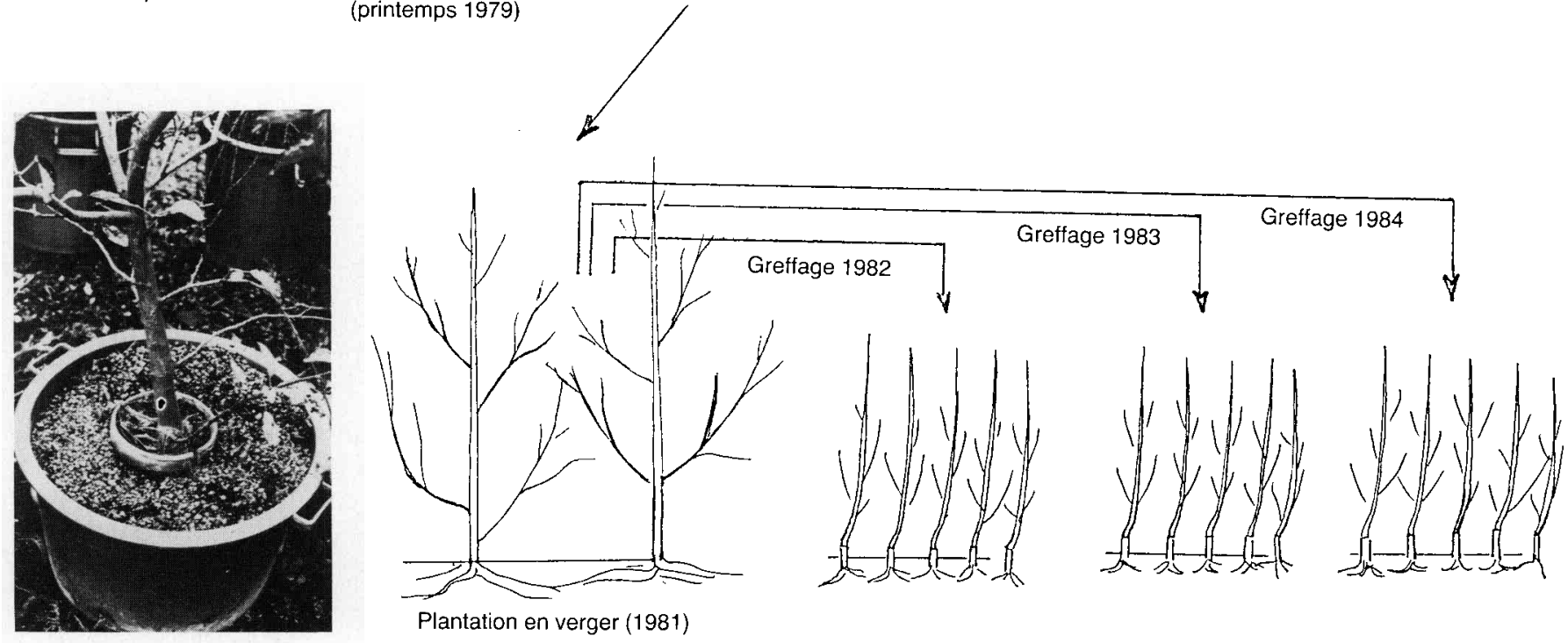

Figure 1

Méthode d'obtention des drageons puis de leur multiplication.

System used to obtain suckers and their propagation in successive nurseries

Golden Delicious et quatre drageons de Stark Golden Spur. La seconde (1981) a porté sur douze drageons de Golden Delicious.

Dans le rang de pépinière, on a alternativement écussonné un bourgon issu de drageon et un bourgeon issu du clone initial (témoin), réalisant ainsi un dispositif couple. Ce dispositif est particulièrement bien adapté à une disposition linéaire. Il permet la comparaison de moyennes de deux échantillons appariés.

A l'issue de la première année de croissance, les scions obtenus ont fait l'objet d'observations sur la vigueur (hauteur et la circonférence mesurée à $20 \mathrm{~cm}$ au-dessus du point de greffe). Les ramifications immédiates, désignées par la suite sous le terme d'anticipés, ont été comptées sur chaque scion. Cinq feuilles successives ont été prélevées dans le tiers supérieur de l'axe primaire pour en mesurer la longueur (L) et la largeur (l).

\section{RÉSULTATS ET DISCUSSION}

Ils sont présentés en trois étapes :

- Les observations en pépinière de la première multiplication des drageons.

- Le comportement des arbres en verger.

- L'évolution des caractéristiques observées lors des campagnes de greffage successives.

\section{A. Observations en pépinière}

Le tableau 1 présente les valeurs des différents paramètres mesurés sur les scions des cinq sous-clones de Golden Delicious, symbolisés par la lettre R. Un test DUNCAN établit les limites de signification au seuil de 5 p. 100 . On remarque que les scions de chaque sousclone ne diffèrent pas du témoin pour la hauteur ou la circonférence bien qu'ils soient généralement plus grands et plus fins, ce qui se traduit par un aspect plus grêle. Par contre, ils en diffèrent presque tous pour le nombre d'anticipés. La taille des feuilles est significativement plus réduite sur les deux dimensions. La forme (rapport L/l) n'est pas modifiée. Les sous-clones manifestent donc des valeurs qui se situent dans le même groupe de signification, avec une réserve pour R5 qui s'en écarte légèrement pour deux caractéristiques (nombre d'anticipés et longueur des feuilles).

De même, les scions des douze sous-clones obtenus l'année suivante sont régulièrement un peu plus grands $(+3 \mathrm{~cm})$ et de calibre un peu plus faible $(-2 \mathrm{~mm})$ que les témoins. Ils ont davantage d'anticipés $(+4)$, les feuilles sont nettement plus petites $(-16 \mathrm{~mm}$ sur la longueur, $-10 \mathrm{~mm}$ sur la largeur), sans que leur rapport soit modifié. De ce fait, nous avons utilisé par la suite, comme nouvelle variable, le produit longueur $\times$ largeur $(\mathrm{L} \times 1)$.

Alors que des quantités importantes de scions de la variété Golden Delicious sont commercialement multi- 
TABLEAU 1

Caractéristiques de croissance des scions des sous-clones $R$ de Golden Delicious.

Growth characteristic's of Golden Delicious maiden tres's originated from suckers (sub-clones $R$ )

\begin{tabular}{|c|c|c|c|c|c|c|c|}
\hline \multirow{2}{*}{ Traitements } & \multicolumn{2}{|c|}{ Scion } & \multirow{2}{*}{$\begin{array}{c}\text { Nombre } \\
\text { d'anticipés }\end{array}$} & \multirow{2}{*}{$\begin{array}{l}\text { Allongements } \\
\text { cumulés }\end{array}$} & \multicolumn{3}{|c|}{ Feuilles } \\
\hline & Hauteur & Circonférence & & & Longueur & Largeur & Rapport L/l \\
\hline Témoins & $119.9 \mathrm{a}$ & $5.80 \mathrm{a}$ & $12,0 \mathrm{a}$ & $372 a$ & $10,83 a$ & $6.73 \mathrm{at}$ & $1,61 \mathrm{a}$ \\
\hline $\mathrm{R} 1$ & $121,6 a$ & $5,17 \mathrm{a}$ & $15,8 b$ & $423 a$ & $9,78 \mathrm{~b}$ & $5,96 \mathrm{~b}$ & $1,64 a$ \\
\hline $\mathrm{R} 2$ & $118,9 \mathrm{a}$ & $5,66 a$ & $15, \mathrm{ib}$ & $354 a$ & $10,08 \mathrm{~b}$ & $5,35 \mathrm{~b}$ & $1,88 \mathrm{a}$ \\
\hline R4 & $123,3 \mathrm{a}$ & $5,54 \mathrm{a}$ & $17,8 \mathrm{~b}$ & $387 a$ & $9.81 \mathrm{~b}$ & $5.86 \mathrm{~b}$ & $1.67 \mathrm{a}$ \\
\hline R5 & $128.5 a$ & $5.92 a$ & $12.8 \mathrm{a}$ & $430 \mathrm{a}$ & $9,11 \mathrm{c}$ & $5.50 \mathrm{~b}$ & $1,66 \mathrm{a}$ \\
\hline
\end{tabular}

L'unité de longueur est le cm.

Les valeurs affectées de la même lettre ne diffèrent pas entre elles au seuil de $5 \%$.

Data followed by the same letter are not statistically significant at $5 \%$ level.

TABLEAU 2

Caractériviques de croissance des scions des sous-clonesr de Stark Golden Spur

Growh characteristics of Stark Goldon Spur maiden wees originated fom suckers (sub-clonex r).

\begin{tabular}{|c|c|c|c|c|c|c|c|}
\hline \multirow{2}{*}{ Traitements } & \multicolumn{2}{|c|}{ Scion } & \multirow{2}{*}{$\begin{array}{c}\text { Nombre } \\
\text { d'anticipés }\end{array}$} & \multirow{2}{*}{$\begin{array}{l}\text { Allongements } \\
\text { cumulés }\end{array}$} & \multicolumn{3}{|c|}{ Fcuilles } \\
\hline & Hauteur & Circonlérence & & & Longueur & Largeur & Rapport L/I \\
\hline Témoins & $90.9 \mathrm{a}$ & $5.09 \mathrm{ab}$ & $9.4 a$ & $154 a$ & $9.77 \mathbf{a}$ & $6,37 \mathrm{a}$ & $1.53 \mathrm{a}$ \\
\hline r2 & $86.9 \mathrm{a}$ & $4.73 \mathrm{~b}$ & $10.2 \mathrm{a}$ & $156 a$ & $8,36 \mathrm{~b}$ & $5,36 \mathrm{~b}$ & $1,56 a$ \\
\hline $\mathrm{rl}$ & $101,9 \mathrm{~b}$ & 5,39 a & $14,0 \mathrm{~b}$ & $246 b$ & $7,83 \mathrm{~b}$ & $4,52 \mathrm{c}$ & $1,73 b$ \\
\hline$r 4$ & $100.9 \mathrm{~b}$ & 5,10 a & $17.1 \mathrm{C}$ & $237 \mathrm{~b}$ & $7,77 \mathrm{~b}$ & $4,56 c$ & $1,70 \mathrm{~b}$ \\
\hline
\end{tabular}

L'unité de longueur est le cm.

Les valeurs affectées de la même lettre ne dilfèrent pas entre elles au seuil de $5 \%$.

Data followed by the same letter are not statistically significant at $5 \%$ level

pliées par greffage chaque année en parfaite conformité, l'observation de quelques drageons fait apparaître des écarts significatifs, en particulier au niveau des dimensions foliaires. Ces différences sont telles qu'elles sont d'ailleurs appréciables à l'œil.

Le tableau 2 présente les valeurs des différents paramètres mesurés sur les scions des quatre sous-clones de Stark Golden Spur, symbolisés par la lettre r. Trois sous-clones sur quatre $(\mathrm{r} 1, \mathrm{r} 3, \mathrm{r} 4)$ diffèrent significativement du témoin pour la hauteur des scions, le nombre d'anticipés, les allongements cumulés, la longueur et la largeur des feuilles et le rapport $\mathrm{L} / \mathrm{l}$. Les valeurs observées pour ces trois sous-clones sont proches de celles trouvées sur les drageons $\mathrm{R}$ traduisant une constitution génétique de type standard. On peut ainsi penser que les racines dont sont issus ces drageons sont également de type standard, ainsi que la couche profonde LIII des boutures de Stark Golden Spur. Les écarts observés, de quelques 10 p. 100 , peuvent être imputés aux contaminations virales.

Par contre, le sous-clone 2 se distingue de ses homologues pour trois caractères (hauteur des scions, nombre d'anticipés et allongements cumulés). Il ne diffère pas du témoin pour ces mêmes caractères. Ce sous-clone r2 nous paraît devoir s'interpréter comme néoformé à partir d'une racine issue exceptionnellement de la couche cellulaire LII porteuse du caractère spur. Toutefois, ce sous-clone $r 2$ se différencie de son témoin spur par les dimensions foliaires plus réduites, sans modification de la forme, dans le même sens que les sousclones $\mathrm{R}$ et $\mathrm{rl}$, r3, r4 se distinguent eux-mêmes du témoin standard.

Les observations en pépinières font donc apparaître deux sources de variations:

- lune peut s'interpréter comme d'origine génétique, les drageons $r 1, r 3, r 4$ provenant de la couche profonde LIII, restée de type standard, tandis que le drageon $\mathrm{r} 2$ est issu d'une racine exceptionnellement formée à partir de la couche LII de type spur. Ceci confirme le caractère en chimère du mutant Stark Golden Spur,

- l'autre, que l'on qualifiera provisoirement de physiologique, affecte l'allure générale des scions. Ce sont les dimensions foliaires qui en rendent le mieux compte. Cette différence se retrouve aussi bien pour les drageons de type standard à l'égard de leur témoin que pour le drageon r2 à l'égard de son témoin spur.

\section{B. Observations en verger}

La circonférence des troncs et les allongements cumulés de l'ensemble des pousses sont rassemblés dans 
le tableau 3. Pour ces deux caractéristiques et, au cours des trois années suivant la plantation, les arbres issus de drageons $\mathrm{R}$ ont un développement tout à fait similaire à celui des arbres témoins. Les trois sous-clones $r 1, r 3, r 4$ continuent de se démarquer très nettement de leur témoin spur, tout particulièrement au niveau des allongements cumulés. Toutefois, on ne retrouve pas pour ces sous-clones les valeurs relevées pour les sousclones $\mathrm{R}$ de Golden, toujours en raison des contaminations virales. L'aspect des arbres du sous-clone $r 2$ reste en tous points identique à celui du témoin spur.

Le tableau 4 fait état de l'évolution du nombre moyen d'inflorescences par arbre au cours des quatre années suivant la plantation. Les arbres $\mathrm{R}$ se distinguent du témoin par une induction florale plus faible au cours des deux premières années, bien que le volume des arbres soit identique (fig. 2). Ils retrouvent une floraison égale au témoin à partir de 1984. L'évolution des arbres r1, r3, $\mathrm{r} 4$ est très similaire aux précédents et les valeurs un peu inférieures enregistrées les années suivantes nous paraissent là encore relever de la différence d'état sanitaire. Les arbres $\mathrm{r} 2$ manifestent aussi les deux premières années une réduction de l'induction florale, significative seulement en 1983. Ils retrouvent le niveau de leur témoin spur à partir de 1984.
Il est de plus intéressant de comparer pour chacun des sous-clones R l'évolution du nombre d'inflorescences au cours des quatre années (fig. 3). Le sous-clone Rl atteint le niveau du témoin dès 1982, R2 l'atteint en 1983, R4 en 1984 et les deux derniers (R3, R5) seulement à partir de 1985. Ceci révèle des différences dans la rapidité des sous-clones à retrouver la floribondité du témoin.

Le poids de fruits récoltés en 1985 figure à la dernière colonne du tableau 4. Ces poids sont strictement parallèles à l'importance de la floraison, traduisant ainsi l'absence d'anomalies graves sur le taux de nouaison des différentes origines d'arbres. L'ensemble des fruits observés s'est révélé conforme aux variétés de départ.

L'évolution sur quatre années de ces différents arbres fait ressortir trois comportements differents par rapport aux deux variétés d'origine:

- les sous-clones $\mathrm{R}$ qui apparaissent comme une Golden Standard pour la vigueur mais dont la floraison est retardée. Le retour au niveau de floribondité du témoin se rétablissant après un à quatre ans,

- les sous-clones $\mathrm{r} 1, \mathrm{r} 3, \mathrm{r} 4$ qui se comportent comme les sous-clones $\mathrm{R}$, aux contaminations virales près,

- le sous-clone r2 qui retrouve, dès 1984, les caractéristiques de son témoin spur.

TABLEAU 3

Evolution en verger des circonférences de troncs et des accroissements cumulés (movenne par arbre).

Growth measurements of trees in orchard (trunk girth and total length of shoots).

\begin{tabular}{|c|c|c|c|c|c|c|c|}
\hline \multirow{2}{*}{ Traitements } & \multirow{2}{*}{$\begin{array}{l}\text { Nombre } \\
\text { d'arbres }\end{array}$} & \multicolumn{3}{|c|}{ Circonférence des trones en } & \multicolumn{3}{|c|}{ Accroissement en longueur cumulés } \\
\hline & & 1982 & 1983 & 1984 & 1982 & 1983 & 1984 \\
\hline Golden témoin & 24 & $7,1 \mathrm{a}$ & $10,9 \mathrm{a}$ & $14.6 \mathrm{a}$ & $23,4 a$ & $121,2 \mathrm{a}$ & $210,1 \mathrm{a}$ \\
\hline Sous-clones R1-2-3-4-5 & 24 & $7,1 \mathrm{a}$ & $11,1 \mathrm{a}$ & $14,8 \mathbf{a}$ & $17,7 \mathrm{a}$ & $117,3 a$ & $199,4 a$ \\
\hline Sous-clones r1-3-4 & 13 & $6,3 b$ & $9,8 \mathrm{~b}$ & $12,9 b$ & $13,8 \mathrm{~b}$ & $76,3 b$ & $138,6 \mathrm{~b}$ \\
\hline Sous-clone r2 & 5 & $5,0 \mathrm{c}$ & $7,5 \mathrm{c}$ & $9,5 \mathrm{c}$ & $1,5 \mathrm{c}$ & $14,9 \mathrm{c}$ & $37,0 \mathrm{c}$ \\
\hline
\end{tabular}

L'unité de longueur est le cm.

Les valeurs affectées de la même lettre ne diffèrent pas entre elles au seuil de $5 \%$.

Data followed by the same letter are not statistically significant at $5 \%$ level.

TABLEAU 4

Evolution en verger du nombre d'inflorescences et poids de fruits récoltés en 1985

(movenne par arbre).

Number of inflorescences per tree in the successive years and total vield of fruits in 1985 .

\begin{tabular}{|c|c|c|c|c|c|c|}
\hline \multirow{2}{*}{ Traitements } & \multirow{2}{*}{$\begin{array}{l}\text { Nombre } \\
\text { d'arbres }\end{array}$} & \multicolumn{3}{|c|}{ Nombre d'inflorescences en } & \multirow[b]{2}{*}{1985} & \multirow{2}{*}{$\begin{array}{l}\text { Poids de fruits } \\
\text { en } 1985(\mathrm{~kg})\end{array}$} \\
\hline & & 1982 & 1983 & 1984 & & \\
\hline Golden témoin & 24 & $8,1 \mathrm{a}$ & $26,4 a$ & $220 \mathrm{a}$ & $582 a$ & $34,2 \mathrm{a}$ \\
\hline Sous-clones R 1-2-3-4-5 & 24 & $2,9 \mathrm{~b}$ & $10,7 b$ & $203 a$ & $566 a$ & $32,6 \mathrm{a}$ \\
\hline Sous-clones r 1-3-4 & 13 & $2,6 b$ & $2,1 d$ & $118 b$ & $435 b$ & $24,7 b$ \\
\hline Sous-clone r2 & 5 & $10,5 \mathrm{c}$ & $6,9 c$ & $51 \mathrm{c}$ & $121 \mathrm{c}$ & $16,3 c$ \\
\hline
\end{tabular}

L'unité de longueur est le $\mathrm{cm}$.

Les valeurs affectées de la même lettre ne diffèrent pas entre elles au seuil de $5 \%$.

Data followed by the same letter are not statistically significant at $5 \%$ level. 


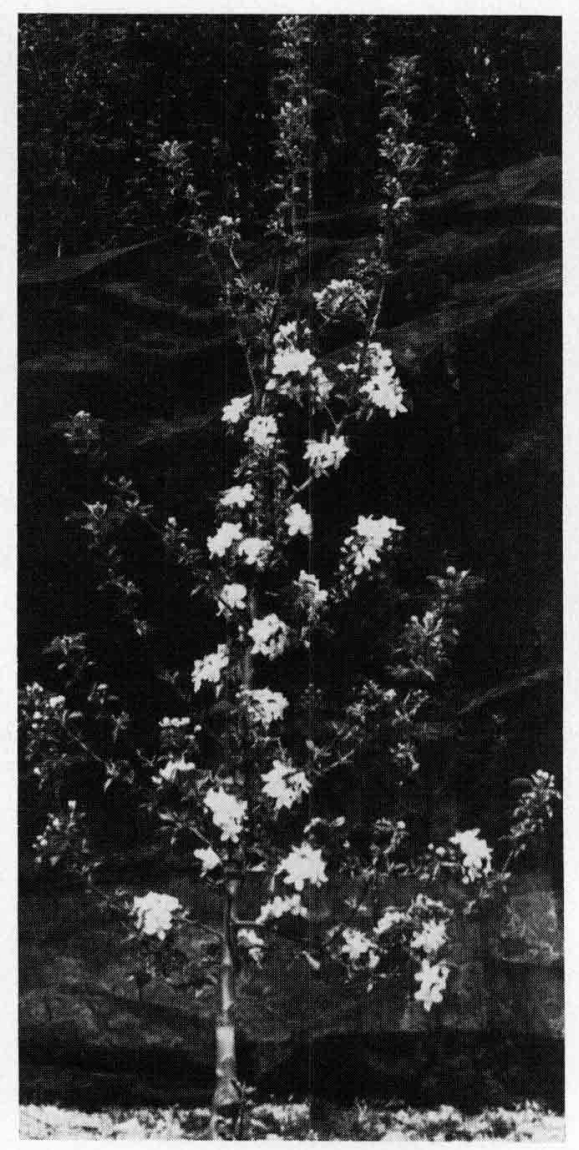

Golden Delicious Témoin

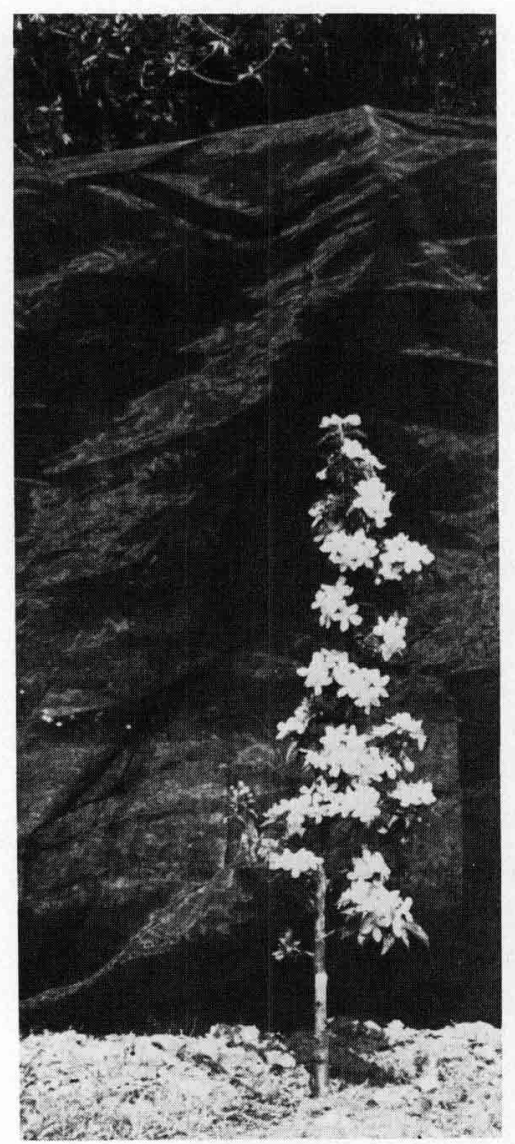

Stark Golden Spur Témoin

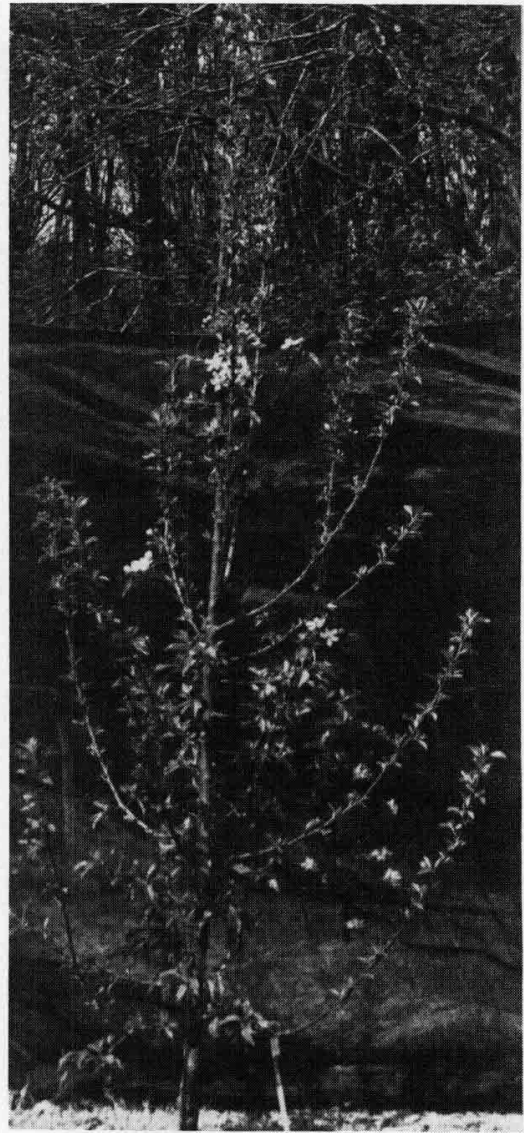

Arbre représentatif d'un sous-clone R de Golden Delicious

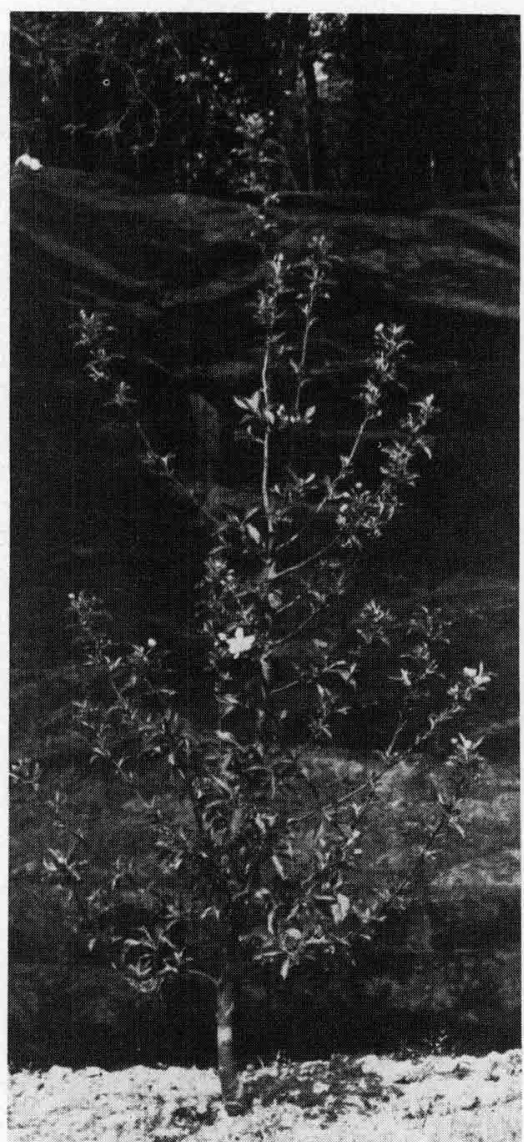

Arbre représentatif d'un sous-clone r1, r3, r4 de Stark Golden Spur.

Figure 2

Aspects des différents tvpes d'arbres issus de drageons de Golden Lelicious et de Stark Golden Spur en mai 1983 (photo I.N.R.A. Angers et Nantes). Size and flowering of different trees obtained from suckers of Golden Delicious and Golden Spur on May 1983 (I.N.R.A. Angers and Nantes photo). 


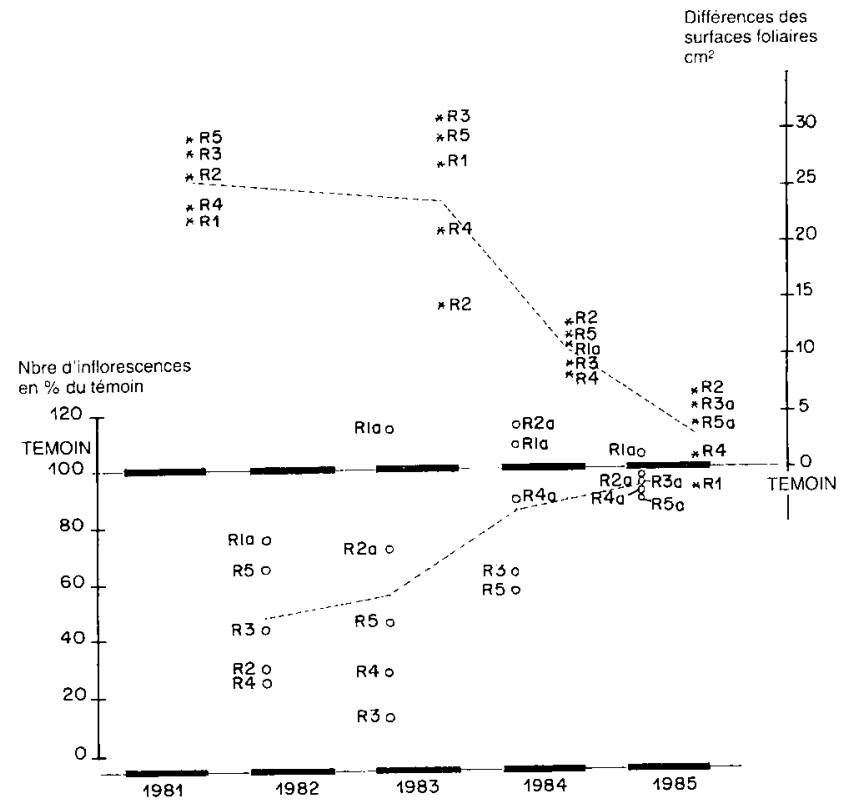

Figure 3

Evolution du nombre dinforescences et des surfaces foliaires des sous-clones $R$.

Inflorescence number and leaf area in $R$ sub-clones.

Les valeurs affectées de la lettre "a ne somt pas significativement différentes du témoin au seuil de $5 \%$.

- Les lignes brisées joighent les moyennes ammuelles.

$V$ altues bearing an " $a$ " did not differ significantly from the control with $P=0,05$.

- Dashed lines indicate annual means.

\section{Evolution dans le temps des caractéristiques foliaires}

La taille des feuilles étant apparue en pépinière comme le principal facteur discriminant entre les drageons et leurs témoins, nous avons cherché à savoir comment cette caractéristique évoluait dans le temps.

Pour ce faire, des greffons choisis d'une façon aussi homogène que possible ont été prélevés sur un arbre de chaque sous-clone, puis écussonnés sur MM 106 en quantité suffisante pour obtenir au moins cinq scions de chaque. Cette opération a été réalisée au cours des campagnes de greffage de 1982, 1983 et 1984 (fig. 1).

Pour chaque campagne, les mesures foliaires ont été effectuées l'année suivante en fin de saison de végétation sur un échantillon de cinq feuilles par scion. Pour l'analyse, nous avons utilisé comme variable unique le produit longueur $\times$ largeur, lequel est étroitement corrélé à la surface foliaire.

Les résultats sont schématisés sur la figure 3 pour les scions des sous-clones R. Au cours des quatre années d'observations, on constate un accroissement des surfaces foliaires des sous-clones vers la valeur des témoins, situation atteinte en 1985 pour R1, R2, R4 et proche de l'être pour R3 et R5. Comme pour la rapidité de mise à fleurs, ces deux derniers sous-clones apparaissent régulièrement plus éloignés du témoin que leurs homologues $\mathrm{R} 1, \mathrm{R} 2, \mathrm{R} 4$. Les sous-clones $\mathrm{r} 1, \mathrm{r} 2, \mathrm{r} 4$ évoluent de la même façon que les sous-clones $R$, mais avec des surfaces foliaires toujours inférieures, en raison des contaminations virales. L'absence de témoin convenable (Golden standard virosée) ne permet pas une étude statistique.
L'évolution du sous-clone $\mathrm{r} 2$ peut, par contre, être comparée à celle de Stark Golden Spur. On constate également une évolution progressive vers des surfaces foliaires égales à celles du témoin, ce niveau étant atteint dès 1983.

\section{CONCLUSION}

Cette étude montre que des variétés de pommier, même anciennes, sont capables de s'enraciner puis de produire des drageons. Deux conclusions peuvent être tirées des observations faites sur ces drageons, l'une d'ordre génétique, l'autre d'ordre physiologique.

D'un point de vue génétique, nous pouvons valablement conclure sur une structure en chimère de la variété Stark Golden Spur. En effet, les drageons de cette variété, qui reflètent la couche profonde LIII, sont du type standard. Par ailleurs, le caractère spur est présent dans la couche de gamétogenèse LII. La structure en chimère de la variété Stark Golden Spur expliquerait par là-même l'instabilité du cultivar constatée dans les vergers commerciaux.

Par contre, le sous-clone $r 2$ doit être interprété comme étant exclusivement constitué de cellules de type spur. DeCOURTYE (1987) a, en effet, montré qu'à partir de chimères colorées, aucun drageon néoformé n'a une structure en chimère. Ce sous-clone de structure génétique homogène représente donc pour l'arboriculteur une amélioration importante pour sa stabilité en culture. Une élimination des agents infectieux a été effectuée par thermothérapie, les indexages de contrôle se sont révélés favorables, ce qui permet de disposer désormais d'un clone de Golden Spur stable et sans virus.

Il reste à comprendre pourquoi l'observation de 144 sous-clones obtenus après irradiation n'ont pas mis en évidence la constitution chimérique de ce mutant (DeCOURTYE, 1970). Il est peu probable qu'aucun retour au type standard ne se soit alors produit. Nous pensons plutôt que les observations succinctes réalisées en pépinière n'ont pas été suffisantes pour mettre en évidence de tels retours.

D'un point de vue physiologique, nous avons pu mettre en évidence des différences entre les drageons régénérés et les variétés d'origine. Les dragcons ont:

- des ramifications plus nombreuses et plus longues.

- une surface foliaire plus faible,

- un nombre d'inflorescences réduit pendant plusieurs années.

Ces différences, très significatives au début de l'étude (1982), s'estompent progressivement jusqu'à disparaître en 1985. Leur nature, comme leur évolution dans le temps, nous paraissent à rapprocher des observations de ZIMMERMAN (1976) sur l'évolution de la juvénilité des semis. L'état atteint par ces sous-clones pourrait se situer dans ce que ZIMMERMAN décrit comme une "zone de transition» entre l'état juvénile d'origine et l'état adulte. Le degré de rajeunissement atteint ne paraît d'ailleurs pas le même pour tous les drageons.

Sur le plan pratique, les caractéristiques de tels arbres risquent aussi de préfigurer le comportement d'arbres issus de culture in vitro technique reconnue pour provoquer également un retour à l'état juvénile. Comparant 
des arbres de Golden spur issus d'in vitro a vec des arbres greffés sur MM 106, ROSATI \& GAGGIOLI (1986) constatent, pour les premiers, un développement végétatif accru et une première floraison retardée.

Des modifications morphologiques du même ordre (forme des feuilles, productivité) ont aussi été constatées sur la vigne après culture in vitro (NOZERAN el al., 1983) et rapportées à un fonctionnement du matériel héréditaire en phase juvénile.
Jusqu'à maintenant, le mode de propagation d'une variété fruitière ne donnait pas accès à ce qu'avait pu être la variété au stade juvénile. La méthode d'obtention de drageons offre une possibilité d'étudier sur un génotype défini les dernières étapes de l'évolution vers le stade fructifère.

\section{RÉFÉRENCES BIBLIOGRAPHIQUES}

Decourtye L., 1969. L'utilisation des rayonnements ionisants pour l'amélioration des arbres fruitiers. C.R. V“ congr. EUCARPIA, Milan 30 sept.-20 oct. 1968. Genetica Agraria, Vol. 33, 166-175.

Decourtye L., 1970. Réflexions sur les possibilités de sélection précoce en amélioration du pommier. Proceedings of the Angers Fruit Breeding Simposium, EUCARPIA, 14-18 sept. 1970, I31-143. Ed. SEI Versailles.

Decourtye L., 1987. Lorigine ontogénique des racines, puis des bourgeons néoformés sur des chimères de poiricr (P1ras communis L.) et de pommier (Malus pumila Mill.). Agronomie, 7 (1), 27-32.

Decourtye L., Lantin B., 1969. Contribution à la connaissance des mutants spurs de pommier; hérédité du caractère. Ann. Amélior. Plantes, 19 (3), 227-238

Decourtyle L., Lantin B., 1971. Considérations méthodologiquss sur lisolement de mutants provoqués chez le Pommier et le Poirier. Ann Amélior. Plantes, 21 (1), 29-44.

Dermen H., 1951. Ontogeny of tissues in stem and leal of cytochimeral apples. Amer. J. Bot., 38, 753-760.

Einset J., 1952. Spontaneous polyploidy in cultivated apples. Proc Amer. Hort. Sci., 59, 291-302.

Faedi W., Rosati P., 1985. Reversion in spur clones of Delicious Apple trees. Acta Hortic., 159, Tree fituits and Nuts breeding, 57-61
Favre J. M., 1977. Formation el entrée en croissance des racines adventives. Comple rendu des séminaires du groupe d'éfude des racines, 4-6 oct. 1977. Angers. Tome 5, 7-36.

Lemoine J., 1986. Les matadies de dégénérescences du poirier el du pommicr. Phyloma Défense des cultures, n³ 380, 22-26.

Nozeran R., Grenan S., Truel P., Favre J. M., 1983. Morphogénèse à partir du stade juvénile de Vitis vimifera L. issu de grainc ou de culture in vitro. Agronomic, 3 (7), 681-684.

Robinson J. C., Schwabe W. W., 1977. Studies on the regeneration of apple cultivars from root cultings. I. Propagation aspects. J. Hort. Sc. 52. $205-220$.

Rosati P., Gaggioli D., 1986. Field performance of micropropagated peach rootstocks and scion cultivars of sour cherry and apples. 192. In Tissue Culure as Plant Production strtem for horticultural crops.

Ed. R. H. Zimmerman and al.

Tilney Basset R. A. E., 1986. Plam chimoras. Ed. Arnold E. London. $199 \mathrm{p}$.

Zimmerman R. H., 1976. Juvenility and flowering of fruit trecs. Acta Hortic., 34. "Grovth regulators in finit production", 139-142. 